\title{
Reliability Estimation of Existing Concrete Bridge Based on Bayesian Dynamic Model
}

\author{
Shuangrui Chen ${ }^{1}$ and Quansheng Yan ${ }^{2, *}$
}

\begin{abstract}
School of Civil Engineering and Transportation, South China University of Technology, Guangzhou, Guangdong, 510640, China
\end{abstract}

\begin{abstract}
It is of great significance to timely and accurately forecast the safety state of the bridge as far as the maintenance is concerned. Bayesian forecasting is a method of deriving posterior distribution in accord with the sampling information and prior information, where real time online forecasting is realized by means of recursive algorithm and the stationary assumption. Bayesian dynamic linear model is created to forecast the reliability of the bridge on the basis of the observed stress information of a bridge structure. According to the observed information, the model created is a superposition of constant mean model and seasonal effect model. The analysis of a practical example illustrates that Bayesian dynamic linear modes can provide an accurate real time forecast of the reliability of the bridge.
\end{abstract}

Keywords: Bayesian dynamic model, forecast, information updating, reliability.

\section{INTRODUCTION}

Conventionally, the researches on the reliability of bridge structure implement reliability parameters to reflect the safety state, not considering the possible changes caused as the time passes on. As a matter of fact, the safety state of a bridge is intensely relevant to the traffic condition, that is to say compared to the late night when the traffic flow is small bridges are under much more pressure in the daytime when the traffic load is heavy. Many bridge collapse accidents are caused by overload traffic, which makes it important to be informed of the instantaneous safety state of the bridge besides the entire bridge structure state in light of a healthy operation [1-4].

Nowadays the reliability forecast and estimation based on the real time observed information on the bridge structure is still on a primary state at home and abroad. Developing fast in recent years, the Bayesian forecasting is a sequential forecast method with dynamic models as the target, the basic idea behind which is to incorporate the forecasters' subjective experience as known conditions, create dynamic models given information on the models, data and prior (the entire distribution information of the unknown parameters) and deduce the Bayesian posterior probability of the state parameters [5-7]. Needless of the stationary assumption, the dynamic models can keep updating as the observed information updates and thus distinguishes itself in the application of real time forecast. Given the many good properties of this method, this article creates Bayesian dynamic linear models on the basis of the observed stress data to verity its application in the reliability forecast of the bridge structure.

\section{THE BAYESIAN DYNAMIC LINEAR MODEL FORECAST OF THE OBSERVED STRESS}

\subsection{The Fundamental Equations of Bayesian Dynamic Linear Models}

The basic idea of Bayesian forecasting is to create dynamic linear models and describe the research by means of observation equations and state equations. Observation equations reflect the way the measured data randomly relies on the current state parameters; state equations reflect the change of the system, representing the internal dynamic change and random intervention [8-10]. Below is the detailed description:

Observation equation:

$$
\begin{aligned}
y_{t} & =\mathbf{F}_{t}^{\prime} \boldsymbol{\theta}_{t}+v_{t} \quad\left(v_{t} \sim N\left[0, V_{t}\right]\right) \\
& \text { State equation: } \\
\boldsymbol{\theta}_{t} & =\mathbf{G}_{t} \boldsymbol{\theta}_{t-1}+\boldsymbol{\omega}_{t} \quad\left(\boldsymbol{\omega}_{t} \sim N\left[\mathbf{0}, \mathbf{W}_{\mathbf{t}}\right]\right)
\end{aligned}
$$

where, $y_{t}$ refers to the observed stress value (scalar); $\boldsymbol{\theta}_{t}$ refers to the n-dimensional state parameter vector; $\boldsymbol{F}_{t}$ refers to $\mathrm{n}$-dimensional regression vector; $\boldsymbol{G}_{t}$ refers to $n \times n$ dimensional state-transition matrix; $v_{t}$ and $\boldsymbol{\omega}_{t}$ refer to stress observational error and state-transition error variable, respectively, $V_{t}$ and $\boldsymbol{W}_{t}$ are corresponding distributional parameters. Usually $\boldsymbol{W}_{t}$ is difficult to be obtained, thus discount factor $\delta(0<\delta<1)$ is introduced here, leading to [10, 11]

$$
\mathbf{W}_{t}=\mathbf{G}_{t} \mathbf{C}_{t-1} \mathbf{G}_{t}\left(\delta^{-1}-1\right)
$$

In the above model, if the prior distribution of $\boldsymbol{\theta}_{t}$ is known or can be derived on the basis of prior information, then according to the observed data calculate the posterior information with the application of Bayesian theorem, the 
result of which will be the prior distribution for the next step. Then it is able to forecast by means of recursion algorithm. Fig. (1) is the flow chart of the algorithm [12, 13].

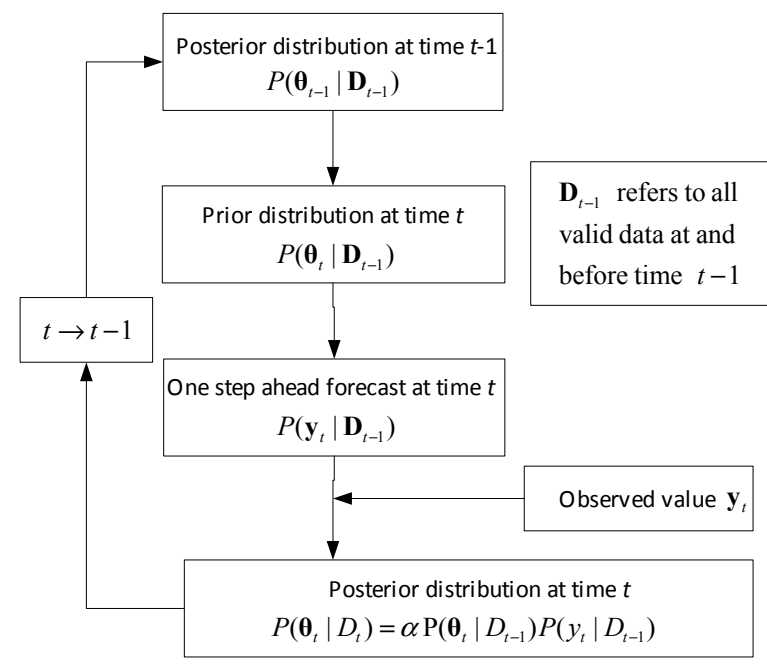

Fig.(1). The recursion algorithm of Bayesian dynamic model [14].

The Bayesian dynamic linear model comprises four elements $\left\{\boldsymbol{F}_{t}, \boldsymbol{G}_{t}, V_{t}, \boldsymbol{W}_{t},\right\}$, noting DLM $\left\{\boldsymbol{F}_{t}, \boldsymbol{G}_{t}, V_{t}, \boldsymbol{W}_{t}\right\}$, among which the formation of $\boldsymbol{F}_{t}$ and $\boldsymbol{G}_{t}$ determines the class of models.

\subsection{The Decomposition and Superposition of Observational Stress Bayesian Dynamic Linear Model}

The linear combination of the independent Bayesian dynamic linear models complies with the superposition principle. The decomposition of complex models into simple component models can simplify the analysis. The load adopted in the bridge reliability calculation is a probability combination of permanent load and variable load, where variable load is intensely relevant to the traffic flow and changes in one day cycle. Thus the observed stress can be decomposed into two parts: a mean value of the stress and a relative cyclic deviation. The former can be described by constant mean model $\boldsymbol{M}_{1}$ and the latter can be described by seasonal model $\boldsymbol{M}_{2}$. Suppose below are the regular representation of $\boldsymbol{M}_{1}$ and $\boldsymbol{M}_{2}[8,15]$ :

$$
\begin{aligned}
& \boldsymbol{M}_{1}:\left\{\boldsymbol{F}_{1 t}, \boldsymbol{G}_{1 t}, V_{1 t}, \boldsymbol{W}_{1 t}\right\}, \text { the state variable is } \boldsymbol{\theta}_{1 t} ; \\
& \boldsymbol{M}_{2}:\left\{\boldsymbol{F}_{2 t}, \boldsymbol{G}_{2 t}, V_{2 t}, \boldsymbol{W}_{2 t}\right\}, \text { the state variable is } \boldsymbol{\theta}_{2 t} .
\end{aligned}
$$

The superposition of $\boldsymbol{M}_{1}$ and $\boldsymbol{M}_{2}$ leads to the load model:

$\boldsymbol{M}:\left\{\boldsymbol{F}_{t}, \boldsymbol{G}_{t}, V_{t}, \boldsymbol{W}_{t}\right\}$, the state variable is $\boldsymbol{\theta}_{t}$.

Where

$$
\begin{aligned}
& \boldsymbol{F}_{t}=\left(\boldsymbol{F}_{1 t}^{T}, \boldsymbol{F}_{2 t}^{T}\right)^{T}, \\
& \boldsymbol{G}_{t}=\operatorname{diag}\left(\boldsymbol{G}_{1 t}, \boldsymbol{G}_{2 t}\right),
\end{aligned}
$$

$$
\begin{aligned}
& V_{t}=V_{1 t}+V_{2 t}, \\
& \boldsymbol{W}_{t}=\operatorname{diag}\left(\boldsymbol{W}_{1 t}, \boldsymbol{W}_{2 t}\right), \\
& \boldsymbol{\theta}_{t}=\left(\boldsymbol{\theta}_{1 t}^{T}, \boldsymbol{\theta}_{2 t}^{T}\right)^{T} .
\end{aligned}
$$

\subsection{Specific Forms of Constant Mean Model and Seasonal Model}

\subsubsection{Constant Mean Model}

The observed stress mean keep changing regularly for a long time. As is mentioned above constant mean model can be applied to analyze it. The specific form of constant mean model is $D L M\left\{1,1, V_{t}, W_{t}\right\}$, the equations are:

Observation equation:

$$
y_{t}=\theta_{t}+v_{t} \quad\left(v_{t} \sim N\left[0, V_{t}\right]\right)
$$

State equation:

$$
\theta_{t}=\theta_{t-1}+\omega_{t} \quad\left(\omega_{t} \sim N\left[0, W_{t}\right]\right)
$$

The remarkable feature of constant mean model is $E\left(y_{t+k} \mid \theta_{t}\right)=\theta_{t}, E\left(\theta_{t+k} \mid \theta_{t}\right)=\theta_{t},(k=1,2, \cdots)$.

\subsubsection{Seasonal Model}

The variable load mainly comprises vehicle load, presenting an obvious periodicity, which makes it suitable to implement seasonal model to study the observed stress mean periodic deviation. The technical term season here describes the time series periodic features. Assume the stress deviation corresponds to mean level is time series $g(t)$ and cycle $p=2 q \quad(q>0), \quad$ then the seasonal vector $\varphi_{t}=\left(\varphi_{t 0}, \varphi_{t 1}, \cdots, \varphi_{t, n-1}\right)^{T}$ at time $t$, leading to the Fourier form of the complete seasonal model:

\section{$D L M\{\boldsymbol{F}, \boldsymbol{G}, \bullet, \bullet\}$}

Where

$$
\begin{aligned}
& \boldsymbol{F}=\left(\boldsymbol{h}_{1}^{T}, \boldsymbol{h}_{2}^{T}, \cdots, \boldsymbol{h}_{q-1}^{T}, h_{q}\right)^{T} \\
& \boldsymbol{G}=\operatorname{diag}\left(\boldsymbol{H}_{1}, \boldsymbol{H}_{2}, \cdots, \boldsymbol{H}_{q-1}, H_{q}\right) \\
& \boldsymbol{h}_{r}= \begin{cases}(1,0)^{T} & r=1,2, \cdots, q-1 \\
1 & r=q\end{cases}
\end{aligned}
$$

(6)

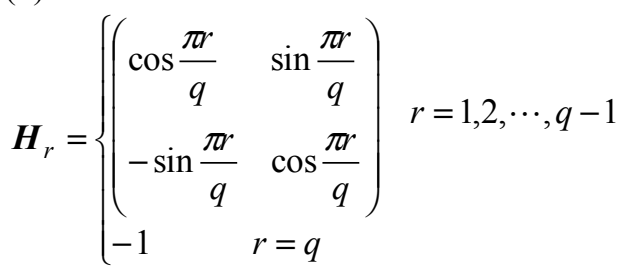

The state variable $\boldsymbol{\theta}_{t}=\left(\boldsymbol{\theta}_{t 1}^{T}, \boldsymbol{\theta}_{t 2}^{T}, \cdots, \boldsymbol{\theta}_{t, q-1}^{T}, \theta_{q}\right)^{T}$, the element of $\theta_{t}$ comprises the Fourier coefficient of $\varphi_{t}$.

From the form of the seasonal model we can know this model is a superposition of $\mathrm{q}$ adaptive component model $D L M\left\{\boldsymbol{h}_{r}, \boldsymbol{H}_{r}, \bullet, \bullet \bullet\right.$. Ignoring harmonic components of less significance by means of significant hypothesis test can simplify the model. For additional simplifying steps please refer to Literature [8]. The form of dynamic model $p=2 q-$ 
1(the cycle is an odd) is similar to that of dynamic model $\mathrm{p}=2 \mathrm{q}$.

\subsection{The Recursion and Correction Algorithm of Bayesian Dynamic Linear Model}

Assume the posterior distribution information of state parameter at time $t-1$ is:

$$
\begin{aligned}
& \left(\boldsymbol{\theta}_{t-1} \mid \boldsymbol{D}_{t-1}\right) \sim T_{n_{t-1}}\left[\boldsymbol{m}_{t-1}, \boldsymbol{C}_{t-1}\right] \\
& \left(V_{t-1}^{-1} \mid \boldsymbol{D}_{t-1}\right) \sim \Gamma\left(n_{t-1} / 2, d_{t-1} / 2\right) \\
& S_{t-1}=d_{t-1} / n_{t-1}
\end{aligned}
$$

Where $\boldsymbol{D}_{t-1}$ is a set of effective information at and before time t-1, $\boldsymbol{D}_{t}=\left\{y_{t}, \boldsymbol{D}_{t-1}\right\}, S_{t-1}$ is the estimation of the observation variance of $V_{t-1}$. Conditional on a large $n_{t-1}$, $T_{n_{t-1}}\left[\boldsymbol{m}_{t-1}, \boldsymbol{C}_{t-1}\right] \rightarrow N\left[\boldsymbol{m}_{t-1}, \boldsymbol{C}_{t-1}\right], S_{t-1} \rightarrow V_{t-1}$, leading to the below recursion relations:

\subsubsection{The prior distribution at time $t$}

$\left(\boldsymbol{\theta}_{t} \mid \boldsymbol{D}_{t-1}\right) \sim T_{n_{t-1}}\left[\boldsymbol{a}_{t}, \boldsymbol{R}_{t}\right]$

where

$$
\begin{aligned}
& \boldsymbol{a}_{t}=\boldsymbol{G}_{t} \boldsymbol{m}_{t-1}, \\
& \boldsymbol{R}_{t}=\boldsymbol{G}_{t} \boldsymbol{C}_{t-1} \boldsymbol{G}_{t}^{T}+\boldsymbol{W}_{t}
\end{aligned}
$$

\subsubsection{The one-step ahead forecast distribution at time $t$}

$$
\left(y_{t} \mid \boldsymbol{D}_{t-1}\right) \sim T_{n_{t-1}}\left[f_{t}, Q_{t}\right]
$$

Where

$$
\begin{aligned}
& f_{t}=\boldsymbol{F}_{t}^{T} \boldsymbol{a}_{t}, \\
& Q_{t}=\boldsymbol{F}_{t}^{T} \boldsymbol{R}_{t} \boldsymbol{F}_{t}+S_{t-1}
\end{aligned}
$$

According to the definition of HPD/the Highest Posterior Density) confidence interval, the HPD confidence interval with a guaranteed rate of $90 \%$ is:

$$
\left[f_{t}-1.645 \sqrt{Q_{t}}, f_{t}+1.645 \sqrt{Q_{t}}\right]
$$

\subsubsection{The posterior distribution for time $t$}

$$
\left(\boldsymbol{\theta}_{t} \mid \boldsymbol{D}_{t}\right) \sim T_{n_{t}}\left[\boldsymbol{m}_{t}, C_{t}\right]
$$

Where

$$
\begin{aligned}
& \boldsymbol{m}_{t}=\boldsymbol{a}_{t}+\boldsymbol{A}_{t} e_{t}, \\
& \boldsymbol{C}_{t}=\boldsymbol{R}_{t}-\boldsymbol{A}_{t} Q_{t} \boldsymbol{A}_{t}^{T}, \\
& \boldsymbol{A}_{t}=\boldsymbol{R}_{t} \boldsymbol{F}_{t} Q_{t}^{-1}, \\
& e_{t}=y_{t}-f_{t}, \\
& d_{t}=d_{t-1}+S_{t-1} e_{t}^{2} / Q_{t}, \\
& n_{t}=n_{t-1}+1 .
\end{aligned}
$$

\subsection{K-Step Lead Time Forecast and Backward Smoothing}

$\mathrm{K}$-step $(\mathrm{k}>1)$ lead time forecast refers to the calculation of the future state distribution on the basis of the information at time t, that is to say calculate $\boldsymbol{P}\left(\boldsymbol{\theta}_{t+k} \mid \boldsymbol{D}_{t}\right)$ on the basis of
$\boldsymbol{P}\left(\boldsymbol{\theta}_{t} \mid \boldsymbol{D}_{t}\right)$. The k-step lead time forecast tells the future change trend of the state variable.

Backward smoothing refers to the past state distribution on the basis of information at time $t$, namely calculate $\boldsymbol{P}\left(\boldsymbol{\theta}_{t-k} \mid \boldsymbol{D}_{t}\right)$ on the basis of $\boldsymbol{P}\left(\boldsymbol{\theta}_{t} \mid \boldsymbol{D}_{t}\right)$. The smoothing analysis provides a review of the evolution of each component of the superposition model, such as the evolution of observed stress mean level. For specific operational formula please refer to Literature [8].

\section{THE RELIABILITY FORECAST BASED ON BAYESIAN DYNAMIC LINEAR MODEL}

This article takes a three-span continuous steel-deck bridge as an example, monitoring the stress of the bottom surface of middle span, and chooses stress as performance function to calculate its reliability. As to this demo, the reliability can be computed with sufficient accuracy by using first-order second-moment method. Equation (15) illustrates the calculation:

$$
\beta=\frac{\mu_{R}-\mu_{D}-\gamma_{M} \mu_{M}}{\sqrt{\sigma_{R}^{2}+\sigma_{D}^{2}+\left(\gamma_{M} \sigma_{M}\right)^{2}}}
$$

where $\mu_{R}$ and $\sigma_{R}$ refer to the mean value and the standard deviation by means of standard calculation, respectively; $\mu_{D}$ and $\sigma_{D}$ refer to stress mean value and the standard deviation caused by permanent load, respectively; $\mu_{M}$ and $\sigma_{M}$ refer to the observed stress mean and the standard deviation, respectively; $\gamma_{M}$ refers to correction coefficient of sensors.

\section{AN EXAMPLE}

Table 1 and Fig. (2) are one-week observed data and corresponding change curve of stress of the middle span bottom surface of the above-mentioned three-span continuous steel deck bridge, respectively.

From Table 1 and Fig. (2), we can see the change of the stress is obviously cyclic, and the whole stress model of can be decomposed into two component models: a constant mean model and a seasonal model.

According to the design information, the formula of the reliability is equation (16):

$$
\begin{aligned}
& \beta=\frac{\mu_{R}-\mu_{D}-\gamma_{M} \mu_{M}}{\sqrt{\sigma_{R}^{2}+\sigma_{D}^{2}+\left(\gamma_{M} \sigma_{M}\right)^{2}}} \\
& =\frac{380-219.1-1.15 \mu_{M}}{\sqrt{29.1^{2}+5.92^{2}+\left(1.15 \sigma_{M}\right)^{2}}}
\end{aligned}
$$

where $\mu_{M}$ and $\sigma_{M}$ are one-step forecast stress mean and one-step forecast stress standard deviation by means of Bayesian dynamic linear model, respectively. It can be seen from the equation that the reliability is only relevant to onestep forecast stress mean $\mu_{M}$ and standard deviation $\sigma_{M}$. Thus the reliability forecast can be realized only on the basis of the mean value of the observed limiting stress derived with the application of Bayesian dynamic linear model.

Having created the model and determining the prior distribution, this article corrects the observed data in the 
Table 1. The observed stress data of the bottom surface.

\begin{tabular}{|c|c|c|c|c|c|c|}
\hline \multirow{2}{*}{ Time } & \multicolumn{6}{|c|}{ Stress at each time (MPa) } \\
\hline & 0:00 & $4: 00$ & 8:00 & $12: 00$ & $16: 00$ & 20:00 \\
\hline Day 1 & 11.95 & 13.62 & 25.68 & 29.19 & 26.70 & 26.40 \\
\hline Day 2 & 16.49 & 8.72 & 25.71 & 28.54 & 24.56 & 26.37 \\
\hline Day 3 & 15.61 & 12.88 & 25.77 & 27.13 & 23.51 & 29.38 \\
\hline Day 4 & 16.85 & 11.63 & 23.33 & 25.43 & 23.07 & 27.95 \\
\hline Day 5 & 16.75 & 9.75 & 22.69 & 26.53 & 28.02 & 27.56 \\
\hline Day 6 & 13.21 & 12.02 & 27.62 & 24.80 & 25.05 & 28.07 \\
\hline Day 7 & 12.68 & 13.21 & 22.68 & 26.04 & 26.24 & 27.00 \\
\hline Day 8 & 14.20 & - & - & - & & - \\
\hline
\end{tabular}

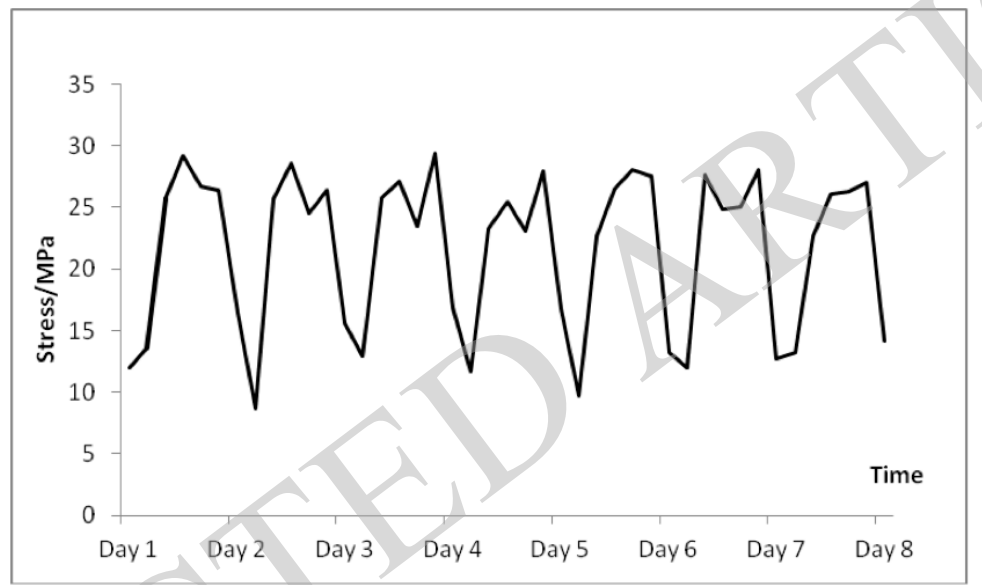

Fig. (2). The change curves of the stress of the bottom surface.

former four days and verifies the forecast of the corrected model with the observed data in the latter three days.

\subsection{Creating Stress Forecast Model}

The first component is a constant mean model $\boldsymbol{M}_{1}:\left\{F_{1}=1, G_{1}=1, \bullet, \bullet\right\}$, describing the trend of the stress change, the prior mean of state vector $\theta_{1}: m_{01}=19$, scale $C_{01}=3.3$.

The second component is the seasonal model $\boldsymbol{M}_{2}$, describing the stress fluctuation around the trend, cycle $\mathrm{p}=6$. The prior mean vector $\boldsymbol{M}_{02}^{*}$ of the seasonal $\varphi$ and prior scale matrix $\boldsymbol{C}_{02}^{*}$ are:

$$
\begin{aligned}
& \boldsymbol{M}_{02}^{*}=(-10.30,-8.64,3.42,6.94,4.44,4.14)^{T} \\
& \boldsymbol{C}_{02}^{*}=\operatorname{diag}(10,10,10,10,10,10)
\end{aligned}
$$

The mean vector and scale matrix of transformed Fourier state vector $\boldsymbol{\theta}_{2}$ :

$$
\begin{aligned}
& \mathbf{m}_{02}=(-7.81,-3.99,-1.68,-3.40,-0.81)^{T} \\
& \mathbf{C}_{02}=\operatorname{diag}(3.3,3.3,3.3,3.3,3.3)^{T}
\end{aligned}
$$

The Fourier seasonal model comprises $p / 2=3$ harmonic components. According to $\mathrm{F}$ distribution test, the former two components are more obvious while the last component can be ignored. The simplification leads to:

$$
\begin{aligned}
& \boldsymbol{m}_{02}=(-7.81,-3.99,-1.68,-3.40)^{T} \\
& \boldsymbol{C}_{02}=\operatorname{diag}(3.3,3.3,3.3,3.3)^{T}
\end{aligned}
$$

Superimpose $\boldsymbol{M}_{1}$ and $\boldsymbol{M}_{2}$, then we get a fivedimensional model:

$$
\boldsymbol{M}:\{\boldsymbol{F}, \boldsymbol{G}, \bullet, \bullet\}
$$

Where

$$
\begin{aligned}
& \boldsymbol{F}=(1,1,0,1,0)^{T} \\
& \boldsymbol{G}=\operatorname{diag}\left(1, \boldsymbol{H}_{1}, \boldsymbol{H}_{2}\right) \\
& \boldsymbol{H}_{r}=\left(\begin{array}{ll}
\cos \pi r / 3 & \sin \pi r / 3 \\
-\sin \pi r / 3 & \cos \pi r / 3
\end{array}\right) \quad(r=1,2)
\end{aligned}
$$

Initial prior information:

$n_{0}=6, S_{0}=9, d_{0}=S_{0} n_{0}$, 


$$
\begin{aligned}
& \left(V^{-1} \mid \boldsymbol{D}_{0}\right) \sim \Gamma\left(n_{0} / 2, d_{0} / 2\right), \\
& \left(\boldsymbol{\theta}_{0} \mid \boldsymbol{D}_{0}\right) \sim T_{n_{0}}\left(\boldsymbol{m}_{0}, \boldsymbol{C}_{0}\right), \\
& \boldsymbol{m}_{0}=(19,-7.81,-3.99,-1.68,-3.40,-0.81)^{T} \\
& \boldsymbol{C}_{0}=\operatorname{diag}(3.3,3.3,3.3,3.3,3.3,3.3)^{T} .
\end{aligned}
$$

Discount factor $\delta=0.9$.

\subsection{No Data K-Step Lead Time Forecast}

Conduct the $k(k=1,2, \ldots, 25)$ step lead time forecast conditional on $\boldsymbol{D}_{\boldsymbol{0}}$ ( no observed data imported) and the result is in Fig.3. The forecasting error is presented in Fig 4.

It can be seen from Fig. (3). The measured data are within the 90\% HPD forecasting interval by and large. However the large and diffuse intervals lead to the uncertainty of the forecast. From Fig. (4), we can see that almost all the forecasting errors are positive, which implies a low forecast value and the calls for the correcting the model with measured data.

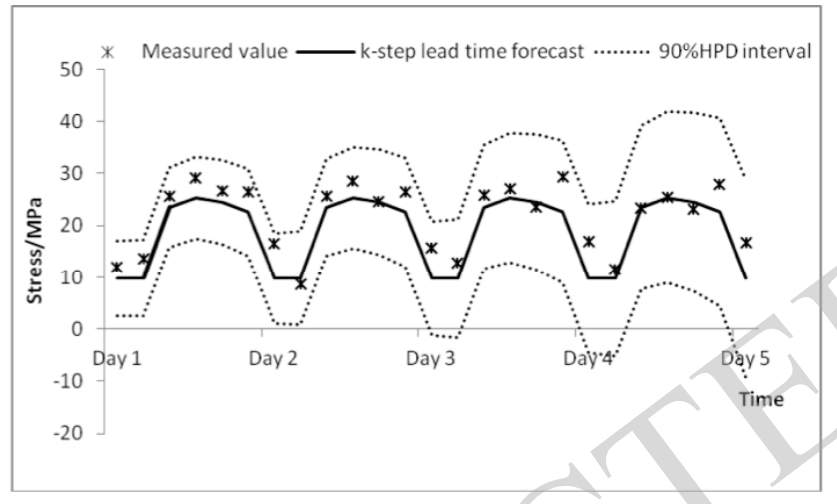

Fig. (3). The k-step lead time forecast conditional on D0.

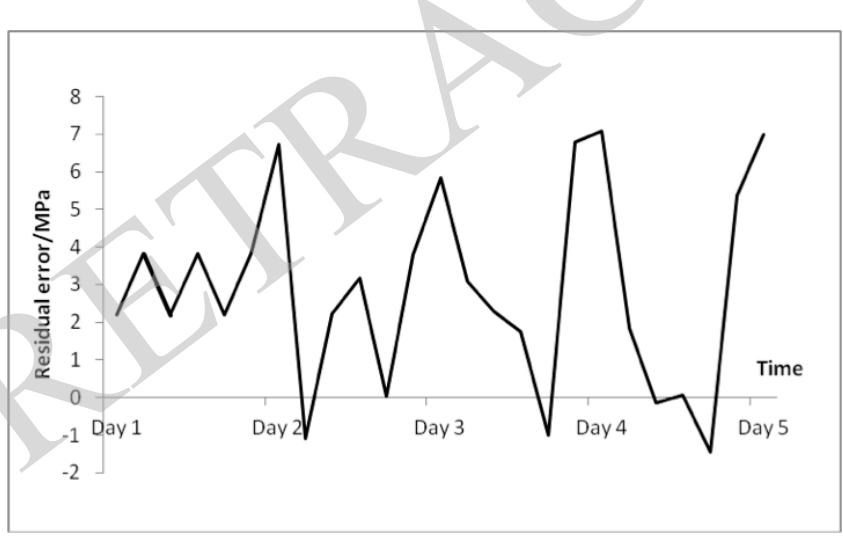

Fig. (4). No data k-step lead time forecast error.

\subsection{One-Step Ahead Forecast}

Import the observed data and correct the model. Fig. (5) is the one-step ahead forecasting. The measured data are mainly covered by the forecast interval. With the increase of observed information, the forecast interval tends to converge, leading to a small uncertainty. The one-step ahead forecast error curve (Fig. 6) fluctuates around 0 and the model matches the measured data well.

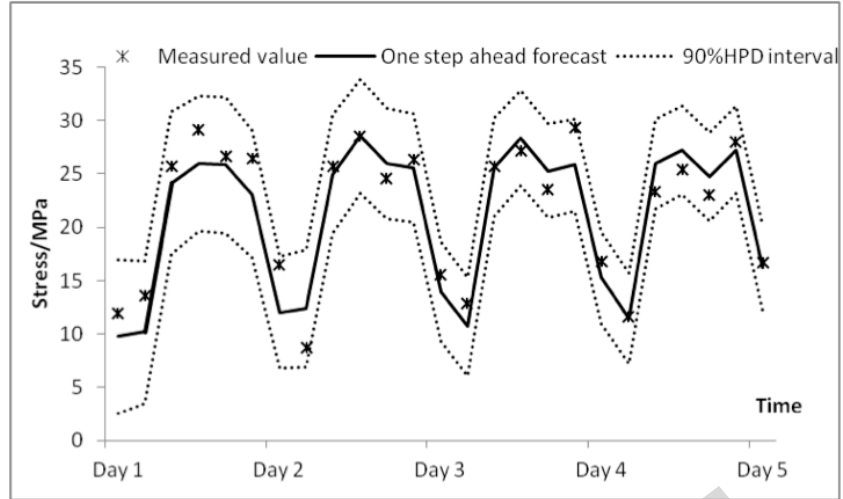

Fig. (5). One-step ahead forecast.

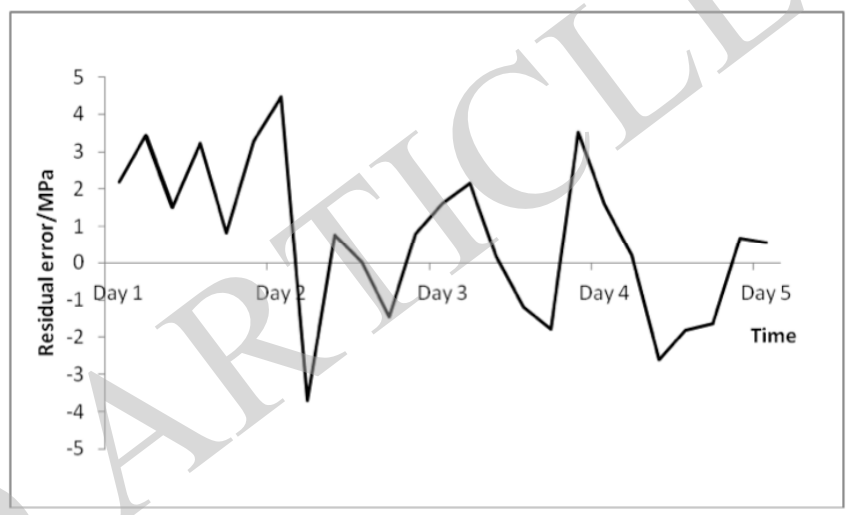

Fig. (6). One-step ahead forecast error.

\subsection{Backward Smoothing Analysis}

Conduct the backward smoothing analysis conditional on D25 (day 5 zero o'clock, 25 observed data imported). Suppose the distribution of state variable $\boldsymbol{\theta}$ is $\left(\boldsymbol{\theta}_{t-k} \mid \boldsymbol{D}_{t}\right) \sim T_{n_{t-k}}\left[\boldsymbol{m}_{t-k}, \boldsymbol{C}_{t-k}\right], \mathrm{k}=1,2, \ldots, 24$, at time t-k; the filter vector $\mathrm{X}$ is five-dimensional, $x_{t-k}=\boldsymbol{X}^{T} \boldsymbol{\theta}_{t-k}$ then according to statistics there is $\left(x_{t-k} \mid D_{t}\right) \sim T_{n_{t-k}}\left(\boldsymbol{X}^{T} \boldsymbol{m}_{t-k}, \boldsymbol{X}^{T} \boldsymbol{C}_{t-k} \boldsymbol{X}\right)$. Thus by creating different $X$ can review the evolution of each component or the combination of components.

Conditional on $\boldsymbol{X}=\boldsymbol{F}$, then $x_{t}$ is the smoothing estimation of the whole model at time $t$. See Fig.7. A comparison of Fig. (7) and Fig. (5) shows the backward smoothing curve is better in matching the measured data than one-step ahead forecast curve and the forecast interval is more converged than that of one-step ahead forecast. That is because during the backward smoothing analysis enough information has been derived, leading to smaller uncertainty.

Conditional on $\boldsymbol{X}=(1,0,0,0,0)^{T}, x_{t}$ refers to the smoothing estimation of the non-seasonal component (namely constant mean model component). See Fig.8. As is presented in Fig. (8), the non-seasonal components mainly keeps at the same level, corresponding to the whole trend of stress change in Table 1. $x_{t}$ is the smoothing estimated value of the seasonal component (namely seasonal model 
component) conditional on $\boldsymbol{X}=(0,1,0,1,0)^{T}$. See Fig. (9). The seasonal components periodically fluctuate around 0 , which corresponds to Table $\mathbf{1}$ where a periodic deviation can be seen between stress and the whole trend. Fig. (7) is the superposition of Figs. $(\mathbf{8}, \mathbf{9})$.

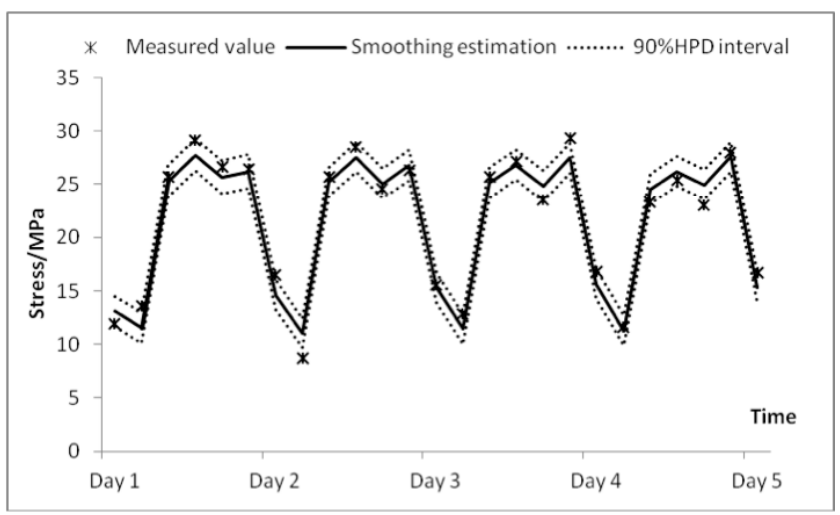

Fig. (7) . The backward smoothing of the stress.

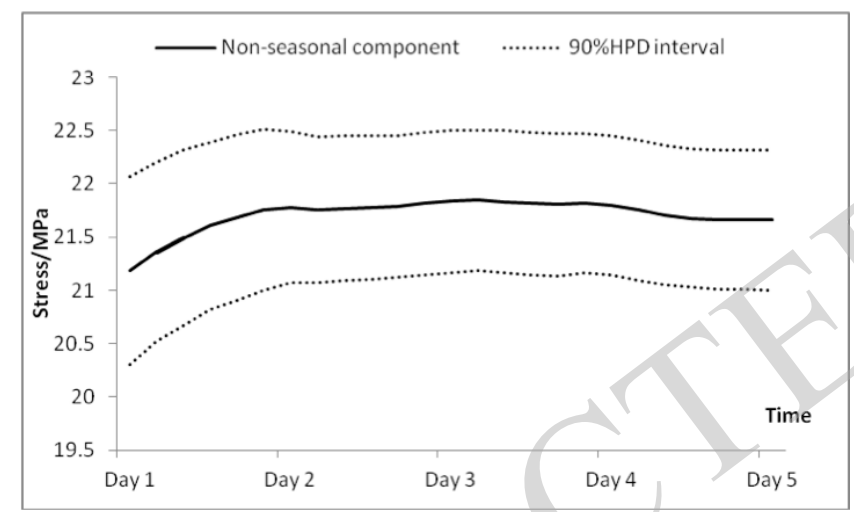

Fig. (8) . The non-seasonal change of the stress.

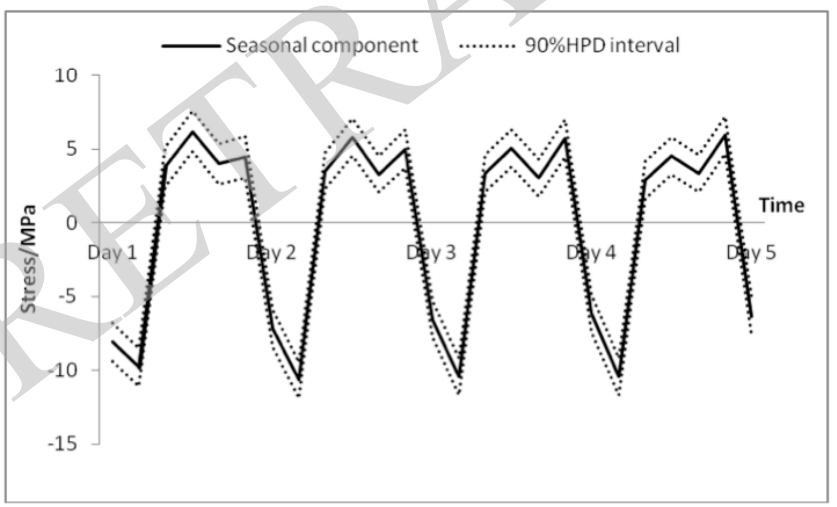

Fig. (9). The seasonal change of the stress.

\subsection{The Verification of the Forecasting Performance of The Dynamic Models}

Fig.10 is the $k(k=1,2, \ldots, 18)$ step lead time forecast on day 5-7 conditional on $\boldsymbol{D}_{25}$. It can be seen from the Fig. (10) that the forecast curve matches the measured data well; the measured data are all covered by the forecast interval and the model show great forecast performance.

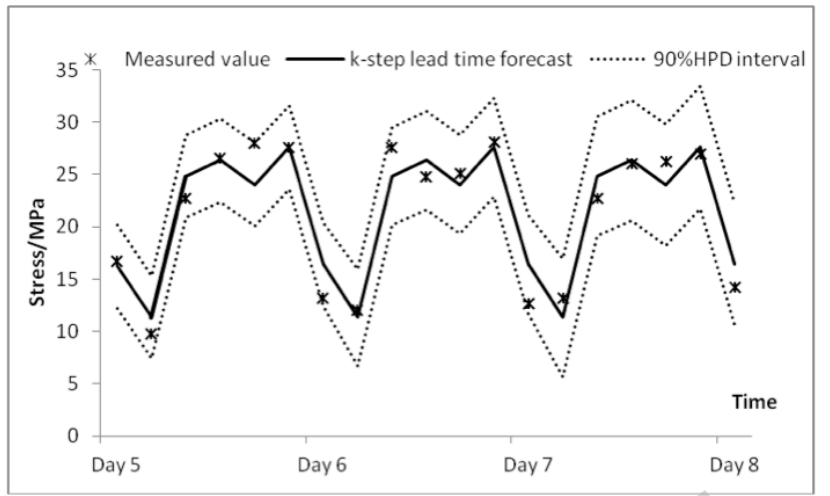

Fig.(10). The k-step lead time forecast conditional on D25.

\subsection{The Bayesian Forecasting of the Reliability}

Fig. (11) is the one-step ahead forecast of the bridge structure reliability utilizing equation (16), and it reflects the real time change. Fig. (12) is the non-seasonal component change conditional on D43. The component basically keeps at the same level, which corresponds to the whole reliability change trend of this demo.

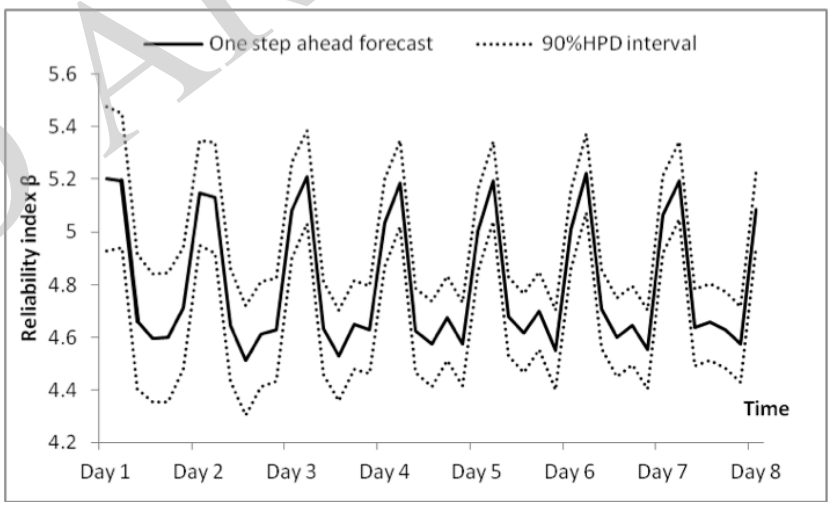

Fig. (11). The one-step ahead forecast of the reliability.

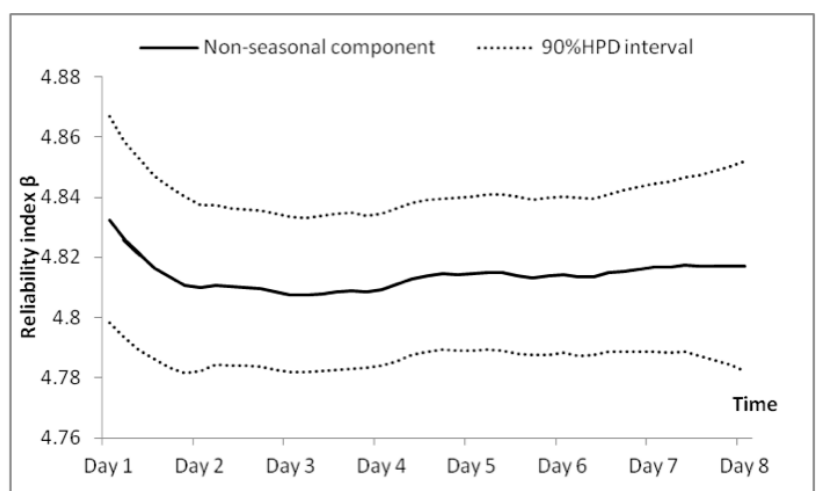

Fig. (12). The non-seasonal change of the reliability.

\section{CONCLUSION}

(1) This example adopts Bayesian dynamic forecast and provides an accurate stress and reliability forecast. This method is recursive Bayesian estimation, making it unnecessary to keep all the data, which saves much memory and realize an online real time forecast. 
(2) Besides the forecast value, the Bayesian forecasting comprises the confidence interval, the width of which determining the uncertainty of the forecast value, making the forecast more instructive.

(3) The Bayesian dynamic linear model created in this article is based on the observed stress data, supposing all the data are normal. As to the inevitable abnormal data occurring in the practice, it is necessary to establish data monitoring system to improve the accuracy. This problem will be further studied later on.

\section{CONFLICT OF INTEREST}

The authors confirm that this article content has no conflict of interest.

\section{ACKNOWLEDGEMENTS}

This work is supported by the Project of National Science Foundation, China (No. 11202080).

\section{REFERENCES}

X. P. Fan, and L. Da-Gang, "Real-time reliability forecast of bridge structures based on multiple BDLMs," Journal of South China University of Technology (Natural Science Edition). vol. 41, pp. 70-75, 2013

[2] X. P. Fan, Time-dependent Reliability Assessment of Concrete Continuous Beam Bridge Based on Real-time Monitoring Information, Harbin Institute of Technology, Harbin, 2010.

[3] X.Y. Sun, H. Pan, H.L. Wang,and J. Wang, "Time-dependent reliability analysis of existing bridges under atmospheric environment," Journal of Zhejiang University (Engineering Science), vol. 46, pp. 643-649, 2012.
[4] Y. Ma, J. Zhang, L. Wang, and Y. Liu, "Probabilistic prediction with Bayesian updating for strength degradation of RC bridge beams," Structural Safety, vol. 44, pp. 102-109, 2013.

[5] G.J. Wei, Y. M. Dang, and C.Y. Zhang, "Foundation settlement probability analysis and prediction based on Bayes dynamic linear model," Science of Surveying and Mapping, vol. 37, pp. 52-53, 90, 2012.

[6] Y. Pang, W. Yuan, G. Shen, and X. Deng, "Reliability analysis of highway bridges based on structural vulnerability analysis," Journal of Harbin Engineering University, vol. 33, pp. 1091-1096, 2012.

[7] J. Wang, W. Deng, and J. B. Zhao, "Short-term freeway traffic flow prediction based on multiple methods with bayesian network," Journal of Transportation Systems Engineering and Information Technology, vol. 11, pp. 147-153, 2011.

[8] M. West, and J. Harrison, Bayesian forecasting and dynamic models, Springer-Verlag, New York, 1997.

[9] X. L. Zhang, F. S. Liu, and C. J. Zhang, Bayesian Dynamic Model and Forecasting, Shandong Science and Technology Press, Shandong, 1992.

[10] S. Russell, and P. Norvig, Artificial Intelligence: A Modern Approach, $3^{\text {rd }}$ ed., Prentice Hall, 2009.

[11] J. M. Nichols, E. Z. Moore, and K. D. Murphy, "Bayesian identification of a cracked plate using a population-based Markov Chain Monte Carlo method," Computers \& Structures, vol. 89, pp. 1323-1332, 2011.

[12] H. Huang, X. Deng, and X. U. Pengpeng, "Bayesian crash prediction model based on a consideration of spatial autocorrelation," Journal of Tongji University (Natural Science), vol. 41, pp. 1378-1383, 2013.

[13] S. D. Bekiros, and A. Paccagnini, "Bayesian forecasting with small and medium scale factor-augmented vector autoregressive DSGE models," Computational Statistics \& Data Analysis, vol. 71, pp. 298-323, 2014

[14] K. P. Murphy, Dynamic Bayesian Networks: Representation, Inference and Learning, UC, Berkeley, 2002.

[15] M. D. Murphy, M. J. O. Mahony, L. Shalloo, P. French, and J. Upton, "Comparison of modelling techniques for milk-production forecasting," Journal of Dairy Science, vol. 97, pp. 3352-3363, 2014

Received: Febuary 08, 2015

Revised: April 12,2015

Accepted: May 12, 2015

(C) Chen and Yan; Licensee Bentham Open.

This is an open access article licensed under the terms of the (https://creativecommons.org/licenses/by/4.0/legalcode), which permits unrestricted, noncommercial use, distribution and reproduction in any medium, provided the work is properly cited. 